\title{
Dual-pedicled conjoined abdominal flap for breast reconstruction in a patient with previous radiation therapy
}

Jun Hyeok Kim, Na Rim Kim, Ye Sol Kim, Deuk Young Oh

Department of Plastic and Reconstructive Surgery, Seoul St. Mary's Hospital, College of Medicine, The Catholic University of Korea, Seoul, Korea
Delayed breast reconstruction is challenging because it requires a large volume and symmetry, and the dual-pedicled deep inferior epigastric perforator (DIEP) flap is used with microvascular augmentation. However, candidate recipient vessels in patients who have undergone radiation therapy may be damaged, with uncertain patency. This report suggests a novel method, the dual-pedicled conjoined abdominal flap, in which a free DIEP flap is combined with a pedicled transverse rectus abdominis muscle (TRAM) flap. A 57-year-old female patient who had undergone modified radical mastectomy and radiotherapy 25 years previously was referred for breast reconstruction. A whole abdominal flap was needed, but supercharged anastomosis was very risky due to calcification and scarring of the internal mammary vessel. Therefore, the thoracodorsal vessels were anastomosed with a free left DIEP flap, which was combined with a right-pedicled TRAM flap. The reconstructed volume was sufficient, and the blood flow was intact. The patient presented a symmetric contour without any complications after 4 months. The dual-pedicled conjoined abdominal flap is reliable for delayed breast reconstruction that requires a large volume and skin replacement, especially in patients with radiation-injured recipient vessels. Even if microscopic anastomosis failure occurs, secondary rescue is made possible by the pedicled TRAM flap.

Keywords Breast reconstruction / Radiation / Free tissue flaps / Pedicled flap

\section{INTRODUCTION}

Breast reconstruction continues to evolve on both the artistic and scientific fronts. The deep inferior epigastric artery perforator (DIEP) flap is considered the gold standard for breast reconstruction and results in patient satisfaction, aesthetically satisfactory outcomes, and favorable donor site morbidity [1].

Received: Aug 13, 2020 Revised: Aug 25, 2020 Accepted: Oct 13, 2020 Correspondence: Deuk Young Oh Department of Plastic and Reconstructive Surgery, Seoul St. Mary's Hospital, College of Medicine, The Catholic University of Korea, 222 Banpo-daero, Seocho-gu, Seoul 06591, Korea

Tel: +82-2-2258-6143, Fax: +82-2-594-7230, E-mail: ohdeuk1234@hanmail.net

Copyright @ 2021 The Korean Society for Aesthetic Plastic Surgery.

This is an Open Access article distributed under the terms of the Creative Commons Attribution Non-Commercial License (https://creativecommons.org/licenses/by-nc/4.0/) which permits unrestricted non-commercial use, distribution, and reproduction in any medium, provided the original work is properly cited. www.e-aaps.org
For delayed reconstruction of the breast, a dual-pedicled DIEP flap is indicated to overcome insufficient hemi-abdominal tissue and extensive radiation effects on the chest wall [2]. The dual-pedicled DIEP flap provides a sufficient skin envelope and subcutaneous fat tissue for the creation of a natural-looking breast with adequate volume to match the contralateral breast [3]. The dual-pedicled DIEP flap requires microvascular augmentation, including turbocharging or supercharging; however, radiation therapy can damage candidate recipient vessels [4], resulting in uncertain patency that may lead to flap failure.

Thus, the present report suggests a novel and effective surgical method, a dual-pedicled conjoined abdominal flap that uses a free DIEP flap combined with a pedicled transverse rectus abdominis muscle (TRAM) flap for volume-demanding, unilateral breast reconstruction, even in patients who have undergone radiotherapy. 


\section{CASE REPORT}

A 57-year-old woman who had undergone modified radical mastectomy and radiotherapy 25 years previously was referred for breast reconstruction. The total radiation dose was 4,256 cGy on her left chest. She was a non-smoker and had no previous history of abdominal surgery. Physical examination revealed a linear, 8-cm-long mastectomy scar and a radiation ulcer with skin thinning on the left breast (Fig. 1A).

A preoperative handheld Doppler examination revealed the left thoracodorsal artery with a clearly audible sound, but the left internal mammary artery (IMA) had a less clear sound, implying uncertain vascular patency. Computed tomography (CT) revealed an intact DIEP on both sides of the abdomen, but the left IMA was indistinct and showed severe calcification (Fig. 1B).

A whole abdominal flap was essential to obtain adequate tissue and a skin paddle. However, supercharged anastomosis with the IMA was thought to be too risky due to its calcification and scarring from previous radiotherapy. Supercharged anastomosis within the thoracodorsal branches was also difficult due to their poor condition. Moreover, turbocharged anastomosis with the thoracodorsal main vessel was not preferred since even partial necrosis of the skin should be avoided to prevent complications in the entire area of irradiated skin. Therefore, for sufficient vascularization, the thoracodorsal artery and vein were prepared for a free DIEP flap. Simultaneously, a combined pedicled TRAM flap was also planned to provide a sufficient skin envelope and soft tissue volume.

With the patient under general anesthesia, the left DIEP of the abdominal flap was dissected to just below the bifurcation site of the external iliac artery and vein, and the right-pedicled TRAM was elevated. Next, the dual-pedicled conjoined abdominal flap was prepared. After the flap was rotated counterclockwise and
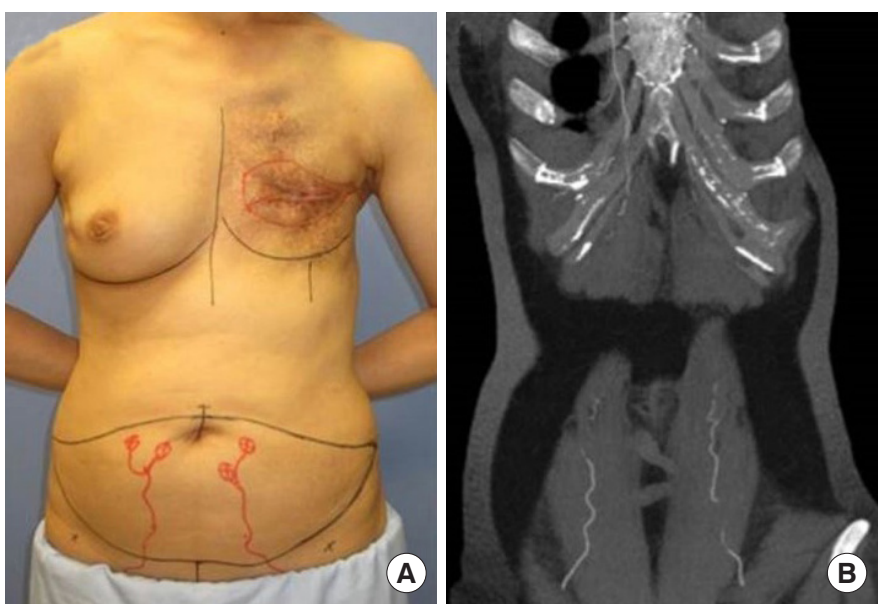

Fig. 1. (A) Preoperative clinical photograph. (B) Computed tomography scan showing scarring, calcification, and uncertain patency of the left internal mammary artery. passed through the subcutaneous tunnel (Fig. 2), the whole abdominal flap was inset (Fig. 3), with intact perfusion of the right hemiabdomen and $3 \mathrm{~cm}$ left of the midline on indocyanine green mapping. Thus, the left DIEP was anastomosed end-to-end with the thoracodorsal vessels. The thin and fragile skin envelope from the radiated chest wall was resected, and no osteomyelitis or chondritis was found around the structure. The whole abdominal flap with a large skin paddle was inset, with a favorable symmetric contour to match the contralateral breast. The reconstructed volume was sufficient, and blood flow was intact on both sides of the flap (Fig. 4). Four months after surgery, the patient presented a symmetric breast contour with no complications.

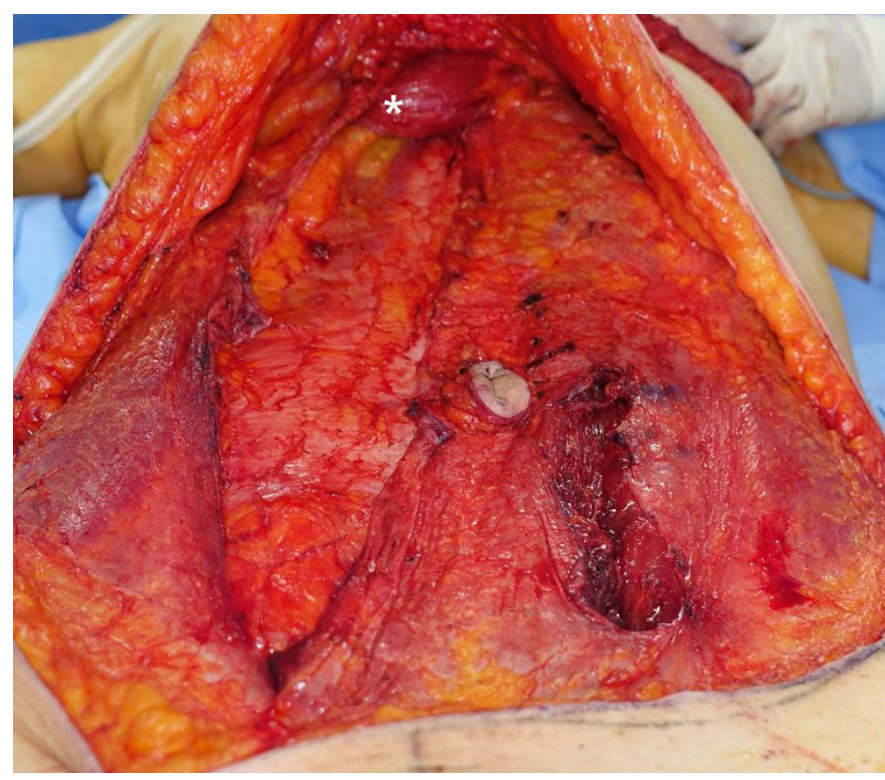

Fig. 2. The dual-pedicled conjoined abdominal flap was rotated counterclockwise and inserted through the subcutaneous tunnel to be inset (white asterisk: pedicled rectus muscle).
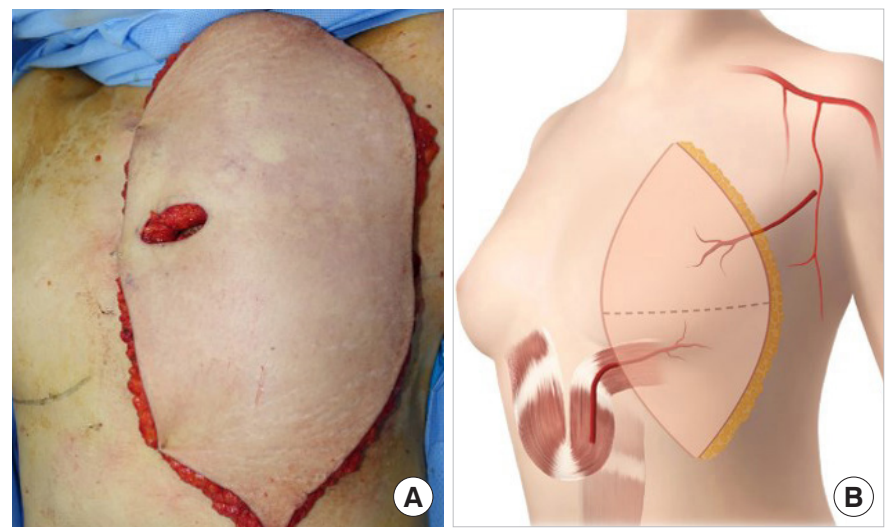

Fig. 3. (A) Insetting of the dual-pedicled conjoined abdominal flap. (B) Schematic diagram of the dual-pedicled conjoined abdominal flap. 


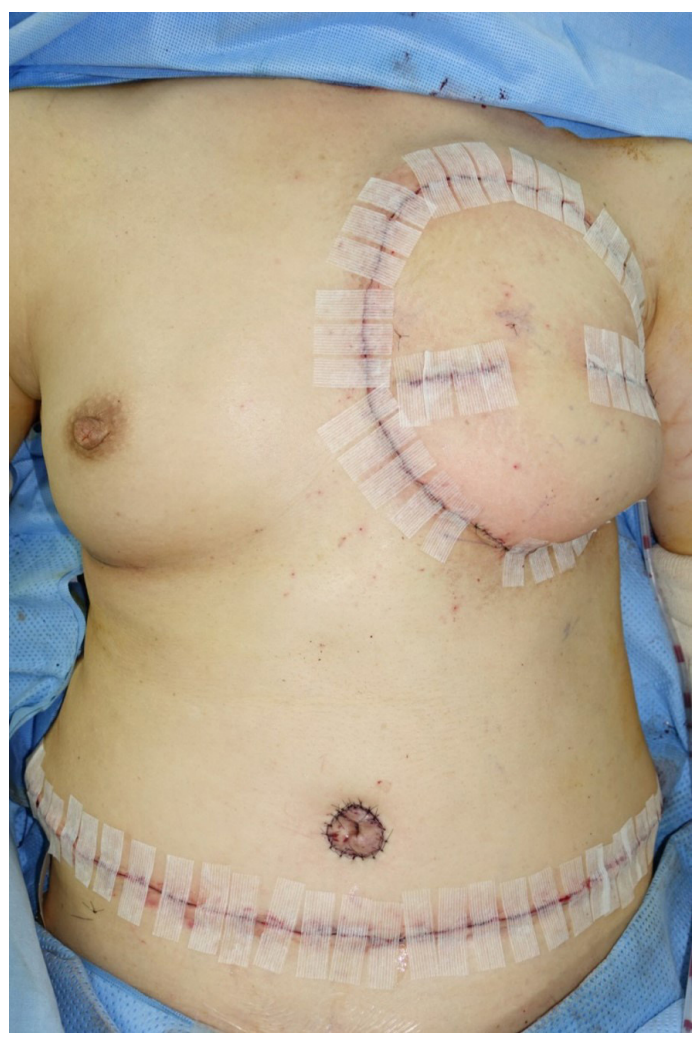

Fig. 4. Immediate postoperative clinical photograph.

\section{DISCUSSION}

Delayed breast reconstruction can be difficult because radiotherapy can cause complications in the chest wall [5], resulting in poor cosmetic outcomes after ischemia, wound breakdown, tissue necrosis, fibrosis, and contracture formation [6]. The dual-pedicled DIEP flap is indicated to overcome insufficient skin and soft tissue volume [2]. The dual-pedicled DIEP flap provides an abundant skin paddle and soft tissue volume, so the patient can be satisfied with a natural-looking breast with adequate volume to match the contralateral breast [3].

Performing a dual-pedicled flap is challenging, and appropriate flap pedicle designs, insets, and perforator must be selected [2]. Although microvascular augmentation is essential for the dual-pedicled DIEP flap to provide ample vascularization, a history of radiation therapy affects recipient vessels, potentially making them unusable [4], with $20 \%$ of internal mammary vessels and $26 \%$ of thoracodorsal vessels being unsuitable for use [7].

Modified microvascular techniques including the use of two pedicles, or the supercharged pedicle flap, and the turbocharged flap have been introduced to enhance the vascular supply to flaps and to decrease the risk of vascular complications [8-11]. A turbocharged abdominal flap is a type of conjoined flap formed by combining the single pedicle with a retrograde loop anastomosis of the contralateral DIEP [8]. However, turbocharging a flap is technically difficult and involves a risk of vascular spasm or thrombosis [9]. Furthermore, a turbocharged flap has only one source of vascular supply, making it difficult to avoid marginal fat necrosis when the absolute amount of blood supply is deficient.

In the patient in the present case, preoperative handheld Doppler and CT revealed uncertain vascular patency of the left IMA due to severe calcification and scarring from previous radiotherapy. Thus, supercharged anastomosis using the IMA was considered hazardous. Moreover, turbocharged anastomosis with the thoracodorsal vessel was avoided due to its poor condition as shown on $\mathrm{CT}$, since even partial necrosis of the skin should be avoided to prevent complications in the entire area of irradiated skin of the chest wall. Thus, the authors had to find a novel technique to eliminate the aforementioned potential risks, and a free DIEP flap using thoracodorsal recipient vessels combined with a pedicled TRAM flap was planned.

Because the dual-pedicled conjoined abdominal flap has a low rate of necrosis [2], the thoracodorsal vessels were prepared as the recipient vessels for the free DIEP flap. Simultaneously, a combined pedicled TRAM flap was planned to provide a sufficient skin envelope and soft tissue volume. Thus, even if microscopic anastomosis failure occurred, secondary rescue would be possible due to the crossed blood flow between the perforasomes from a reliably pedicled TRAM flap.

Some studies have also described supercharged anastomosis of an ipsilateral DIEP flap combined with a pedicled TRAM flap to avoid delayed arterial and venous insufficiency $[10,11]$. Although this technique is somewhat similar to that presented herein in that both involve conjoined flaps made by combining a pedicled TRAM flap with a microvascular anastomosed flap, the crucial difference is whether or not the source vessels come from both sides beyond the midline of the abdominal flap. If both source vessels are on one side, they will be able to provide a rich blood flow to the hemi-abdominal flap, while, as in the present report, if the blood supply comes from both sides beyond the midline, it will be possible to obtain a sufficient skin paddle and soft tissue from the bilateral abdominal flap.

In conclusion, the dual-pedicled conjoined abdominal flap, comprising a free DIEP flap combined with a pedicled TRAM flap, is reliable for delayed breast reconstruction that requires a large volume and skin replacement, especially in patients with radiation-injured recipient vessels. Even if microscopic anastomosis failure occurs, secondary rescue is made possible by the pedicled TRAM flap.

\section{NOTES}

\section{Conflict of interest}

No potential conflict of interest relevant to this article was reported. 


\section{Ethical approval}

The study was approved by the Institutional Review Board of Seoul St. Mary's Hospital (IRB No. KC20ZISI0686) and performed in accordance with the principles of the Declaration of Helsinki.

\section{Patient consent}

The patient provided written informed consent for the publication and the use of her images.

\section{ORCID}

Jun Hyeok Kim

Na Rim Kim

Ye Sol Kim

Deuk Young Oh

$$
\begin{aligned}
& \text { https://orcid.org/0000-0003-4657-2090 } \\
& \text { https://orcid.org/0000-0002-6046-4566 } \\
& \text { https://orcid.org/0000-0003-3970-9212 } \\
& \text { https://orcid.org/0000-0003-3499-1554 }
\end{aligned}
$$

\section{REFERENCES}

1. Hamdi M, Khuthaila DK, Van Landuyt K, et al. Double-pedicle abdominal perforator free flaps for unilateral breast reconstruction: new horizons in microsurgical tissue transfer to the breast. J Plast Reconstr Aesthet Surg 2007;60:904-12.

2. Cho MJ, Haddock NT, Teotia SS. Clinical decision making using CTA in conjoined, bipedicled DIEP and SIEA for unilateral breast reconstruction. J Reconstr Microsurg 2020;36:241-6.

3. Koolen PG, Lee BT, Lin SJ, et al. Bipedicle-conjoined perforator flaps in breast reconstruction. J Surg Res 2015;197:256-64.
4. Tyrell R, Leong RY, Sathyanarayana SA, et al. Using a single internal mammary artery as retrograde and antegrade flow for bilateral deep inferior epigastric artery perforator reconstruction: a case report. Int J Angiol 2016;25:e87-8.

5. D'Souza N, Darmanin G, Fedorowicz Z. Immediate versus delayed reconstruction following surgery for breast cancer. Cochrane Database Syst Rev 2011;(7):CD008674.

6. Javaid M, Song F, Leinster S, et al. Radiation effects on the cosmetic outcomes of immediate and delayed autologous breast reconstruction: an argument about timing. J Plast Reconstr Aesthet Surg 2006;59:1626.

7. Temple CL, Strom EA, Youssef A, et al. Choice of recipient vessels in delayed TRAM flap breast reconstruction after radiotherapy. Plast Reconstr Surg 2005;115:105-13.

8. Semple JL. Retrograde microvascular augmentation (turbocharging) of a single-pedicle TRAM flap through a deep inferior epigastric arterial and venous loop. Plast Reconstr Surg 1994;93:109-17.

9. Cohn AB, Walton RL. Immediate autologous breast reconstruction using muscle-sparing TRAM flaps with superficial epigastric system turbocharging: a salvage option. J Reconstr Microsurg 2006;22:153-6.

10. Vosburg RW, White MJ, Heckler FR. Supercharging of delayed pedicled transverse rectus abdominis myocutaneous flaps, is it a viable option? Microsurgery 2015;35:204-6.

11. El-Mrakby HH, Milner RH, McLean NR. Supercharged pedicled TRAM flap in breast reconstruction: is it a worthwhile procedure. Ann Plast Surg 2002;49:252-7. 